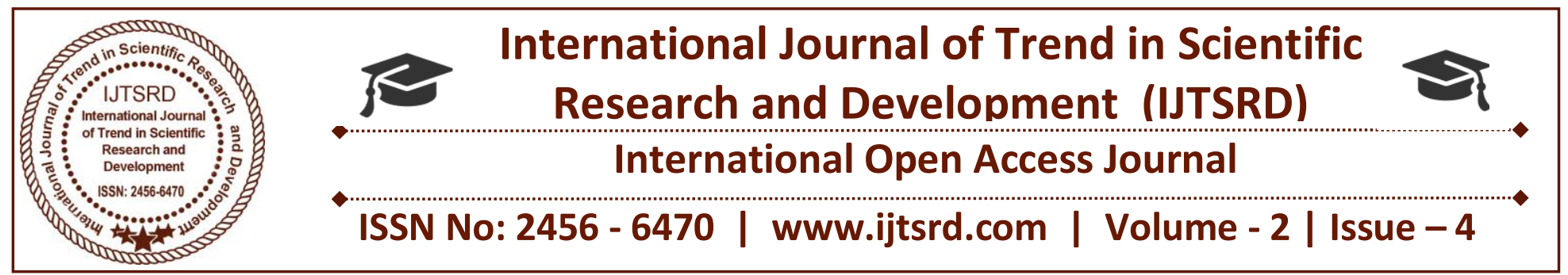

\title{
Segmentation of Front Viewed Single Phase Diamond
}

\author{
${ }^{1}$ Yash Jain N, ${ }^{2}$ T.P.Deepa, ${ }^{2}$ Riya Nayan Shah, ${ }^{3}$ Kajal Jain \\ Computer Science \& Engineering, \\ School of Engineering \& Technology, Jain University, Bangalore, India
}

\begin{abstract}
The estimation of Quality of diamond plays a very important role in finding its price and add a market value. Quality of diamond is being estimated in the market today by just looking into the diamond thought naked or with a lens, but the quality estimated in this way is less accurate and varies from person to person in which the consumers are put into confusion whether the diamond is of good quality or not. It is sometimes difficult to identify whether the diamond is real or not. If we use a computer aided system then the estimation of quality of diamond can be estimated accurately and quickly. Thus, paper aims to develop such computer aided system which will estimate the quality of diamond based on Gemological Institute of America(GIA) standards.
\end{abstract} Keywords: Diamond, GIA Standard, Quality,
Originality

\section{INTRODUCTION}

Most of the diamonds are polished in India and it estimates about more than $80 \%$ of the world's diamonds. Surat is estimated to be worth Rs90, 000 crore (\$13.2 billion) of diamonds and employs over two million people [16]. Nowadays the synthetic diamonds perfectly imitates the real ones. Hence detecting the quality of any diamond through naked eye has become quiet tedious and a difficult process. To prevent such malpractices, there are now large machines built that checks the quality of the diamonds. But such machines aren't quiet portable and cost effective[1].

Therefore this paper now aims at building an application that helps in detection of the quality of all unstudded diamonds, hence solving the limitations of portability and cost.
Well cut diamonds, which are the most expensive types of diamonds, have fine and sharp cuts in them which signifies the proportions, finishing, polishing and the symmetries present in the gem[2].

Using these properties, one can easily distinguish between well cut and poorly cut diamonds. Hence the latter one is sold at premium while discounts are given on the poorly cut gem.

Color is one of the major characteristic that any diamond possesses. The purest diamond is always colorless. But still in certain cases slight tones of yellow or brown patch of color is seen on the colorless diamonds[2].

Diamonds are rated in alphabetical orders based on the color with D awarded to the totally colorless diamonds and $\mathrm{Z}$ awarded to the heavily tinted ones by the Gemological Institute of America(GIA)[2].

The color difference cannot he differentiated with the naked eyes. Diamonds that have the grade $\mathrm{N}$ or above have tints that are visible to human eyes. The ones having grade $\mathrm{K}$ or below still have tints but are beautiful if they have sharp cuts present on them[2][7].

As the grades move below $\mathrm{S}$, the tints on the diamond starts becoming more intense. Diamonds having pink, blue, brown, yellow, orange, green and red as their combinations are quite popular in use. While yellow being the naturally occurring color in a diamond; white, red, blue and green are the ones which are quiet rare types. White diamonds, Black Diamonds, Blue diamonds, Brown diamonds, Yellow diamonds are the coloured diamonds by the sellers [17].The second most significant characteristic of a pure diamond is its size.Size of a diamond determines its value in the market as the prices of all diamonds in the market exponential depends on its size[3]. 
A diamond is always measured in terms of "carats". Normally, 1 carat is equal to 200 milligrams. Carats usually is subdivided into points because most of these diamonds are sold with the weight lesser than 1 carat in the market.

100 points makes a single carat . Hence a "75 point diamond" is said to be weighed as $3 / 4$ carat.

Therefore, $\quad 1$ carat $=200 \mathrm{mg}=100$ points.

The quality of the diamond is not measured by the carat weight as carat approximately denotes how big the size of diamond might be.

Large diamonds are very rare to be found in mines when compared to the smaller ones. Hence they are much more valuable. In smaller ones, Diamonds weighing $0.50-0.75$ are popular in terms of its value and size[3][7].

\section{LITERATURE SURVEY}

Kirtilals, a premier diamond jewellery retail chain, has come up with a portable solitaire laboratory, clients can now walk into the local showroom and check the properties of diamonds, identifying the cut and assessing the true value of their possession. [8]

D-guard is a diamond testing machine by Sparrow Technology, Surat.It is a machine for separating synthetic diamonds like CVD, or HPHT from Natural diamonds in bulk quantities. [8]

Some of the popular diamond testers are:

The Ceres Diamond Tester: it consists of two semiconductors called the thermistor that has specific characteristics with a huge amount of sensitivity along with a minute copper tip on its holder. The tip of this tester is heated and applied on the diamond. This device is quiet accurate because of its sensitivity in temperature which also makes it very reliable[8].

The Rayner Diamond Tester: this British designed tester possesses same attributes to the latter one but is more expensive than it. Erum Queshi, who is the author of "Differentiating Diamond an its limitations" has stated that this type of tester is majorly used by the traders who are completely into diamond trades as their living wholely depends in this industry and buying such a costly tester is just a small thing for them[8].

The GIA's (Gemological Institute of America): This grading institute of diamond has developed a unique tool called the Diamond pen, which works on certain affinities that diamonds express such as grease and greasy liquids hence differentiating the pure diamonds from the synthetic ones. This tool contains a greasy liquid instead of ink that leaves behind visible marks on a normal diamond facet but beads up when put on any other kind of stone without leaving behind any mark[8].

Fluorescence Diamond Testing: This testing is done using the long wave ultraviolet light. Every diamond has certain amount of fluorescence present in them which is measured on a scale of one having little amount or no fluorescence to five having the strongest amount present in the diamond. Every diamond falls in this range. But when it comes to jewellery having multidiamonds, it is very rare to measure fluorescence in many diamonds in the jewellery. It is usually found that all the diamonds present in the jewellery won't have same level of fluorescence. Such consistency in measure is present only in synthetic diamonds.

It is actually being misunderstood that UV light can be used to test a diamond. This is not at all true. Diamonds just fluoresce randomly. UV Light just gives indications when the diamonds are being differentiated. This is done by viewing the stone on a completely black background in a complete dark room with only a long wave UV lamp being present as the source of light in the room. It is observed that pure colorless diamonds glow more (about 40\%) under a long wave UVlight but would glow little under short wave light. But usually synthetic diamonds show opposite reaction by glowing less in the long wave and more under short wave[8].

Moissanite: These re manmade gems which was invented in the 1990s. Moissanite looks exactly like a diamond to any normal human. It is found as a small black crystal of silicon carbide or is found as small platelets which are green in color. This synthetic diamond is the exact imitation of a real diamond and registers DIAMOND on any diamond testers. The size of this stone is less than $1 \mathrm{~mm}$ and cannot be cut. This gem gets it name from a French chemist, Henri Moissanite who found the mineral in the year 1893, in a meteorite crater in Arizona. But on a patent published in 1998 called "translucent silicon carbide of a single polytype that are grown in a furnace sublimation system" it was named as "silicon carbide gemstones"[9].

Moissanite Tester and Type II Diamonds: the testers for moissanite measures the electrical conductivity present through the stone as natural diamonds are not electrical conductive. But Type II 
diamonds which are rare kind of stones have a very unusual chemical component that contains boron, hence making the diamond electrically conductive which registers itself to be mossanite on the diamond tester. Using these diamonds, anxious retailers dupe their customers as it is really impossible to differentiate these synthetic diamonds from the real ones. Model SDS is a tester that checks for the type II synthetic diamonds but this tester is an item which is used by only special cases[9].

Moissanite Testers and UV (Ultra Violet light): UVlight along with a Moissanite or multi testers shows a difference when tested on a moissanite. This was first discovered by one of the gemmologist who stated that, UVlight does makes a difference when it comes to electrical conductivity. This is proven according to the laws of quantum physics. When he worked on the wavelength of the UVlight, he found that all the wavelengths of this light shows the difference. It even works on certain moissanites on which even the Moissanite testers sometimes fails[9].

Diamond Tester or Multi-Tester: Real diamonds are differentiated from the non-diamonds using a diamond tester. But the man made moissanite stone is an exception which will register as "diamond" itself on the tester.

When it comes to a multitester, it helps you to test whether the gem being tested is a diamond or a nondiamond or a moissanite. The exterior model helps to differentiate rubies and saphires too.

Moissanites have become a lot popular with mossanite jewellery becoming more and more popular[9].

\section{METHODOLOGY}

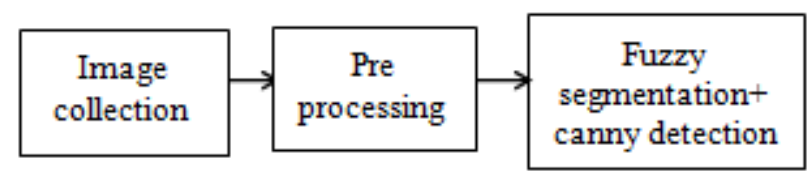

Figure 1: Segmentation of diamond

\section{Image collection}

Images of diamond are taken from infrared cameras that are kept in a dark pallet on the weighing machine and these images are fed to the upcoming image processing.

\section{Pre-processing}

Since the images that are selected as the input image, it is been made sure that the background is made white. The diamond image which is taken is been viewed from a single phase. Therefore not much of image pre-processing is done.

\section{Segmentation:}

The segmentation phase involves separating the diamond (Region of intetest) from the background. Fuzzy segmentation and Canny edge detection was used. Images choosen for this work consists of single diamond stud from top view with white background. The steps followed are as follows-

Step 1: Image is clustered in the based on fuzziness parameter, stopping condition and feature space. Apply this clustering for every pixel in a image. Lets pixel matching with feature space be

Step 2: For every pixel of interest pij, identify c

Cluster $(\mathrm{Cm})$ to which it belongs closely. Find other pixel if it is related to pij, if so, include it into Cluster $(\mathrm{Cm})$.

Step 3: Map Cluster $C_{m}$ into Segmentd $S_{m}$. hence if pixel pij belongs to cluster $\mathrm{C}_{\mathrm{m}}$ then add to segment $\mathrm{Sm}$, otherwise create a new cluster and segment.

Step 4: if there are multiple clusters with same features then merge them into single segment. Apply this to all pairs of clusters. Identify and marks the boundary pixels of all segments using Canny edge detection.

\section{Feature Extraction}

The following features helps to estimate the quality of diamond.

\section{COLOR-}

The color of the diamond is been found before the image of the diamod is been converted into binary or greayscale image by using color based segmentation.

No two diamonds in this world is same so the color of each diamond will have a small change in the color scale. So to find the color of the diamond should be looked from diferent angles inorder to get the correct color grade of the diamond.

The RGB color values are been extracted from the image and compared with the RGB fixed values. Thus by this the color of the diamond is been estimated of the diamond.

\section{CLARITY-}

Natural diamonds are formed due to extreme heat and pressure that is exposed on the carbon present deep inside the earth. This whole procedure results in 
"inclusions" and "blemishes" on the gem. Clarity of diamonds is the major characteristic to determine any diamond's quality. With this characteristic, other major attributes of diamonds such as size, relief, positions etc are determined hence affecting the overall appearance of the stone[10].

\section{CUT-}

When it comes to a real diamond, it is always in a perfect shape as it is sharply cut into a perfect shape which gives an excellent to it. But it is not an easy task to cut a diamond into a perfect shape. Any diamond can only be sharply cut when it has clean and sharp facets which are perfectly symmetric. The real diamond can be cut into a perfect propotions from both the sides of it's girdle. As we talk about the sharp faces on a pure diamond, it is known that most of the real diamonds have atleast 58 clean faces on them. These clear facets are formed on a diamond with the help of a "turntable". This tool is made of porous iron which is coated with the dust and oil of diamond. First, the diamond that has to be cut into facets, is set into a holder and then is held against the turntable. It is then revovled around in a high speed and sharp cuts are made on them. [11].

\section{CARAT WEIGHT-}

Diamonds without inclusions and blemishes are really rare, while most characteristics can be only seen when magnified. The weight of the diamond is always measured in the form carat. The word carat is originated from the word "carob" seed which is the original unit of measure for diamond traders. The price of diamond increases with the increase in the size of diamond (in carats). Larger the diamond, greater is the carat and hence making the diamond much rarer[10][12].

This 4 C's will estimate the quality of diamond after the image processing is done.

\section{RESULTS}

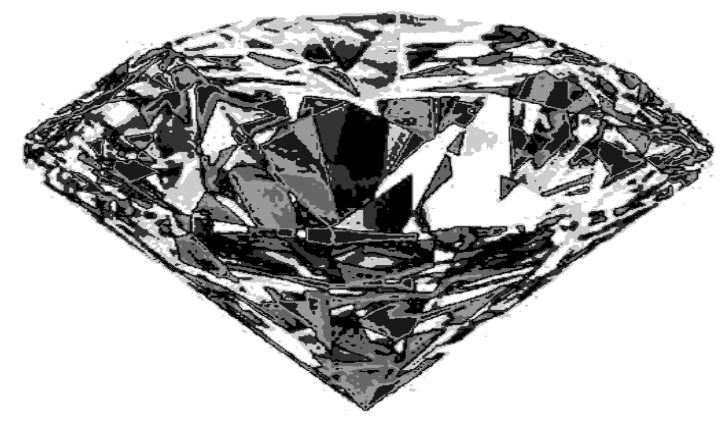

Figure 2: Segmented Image of a diamond

In the above image the diamond image is segmented into its Edges using canny and fuzzy edge based segmentation.

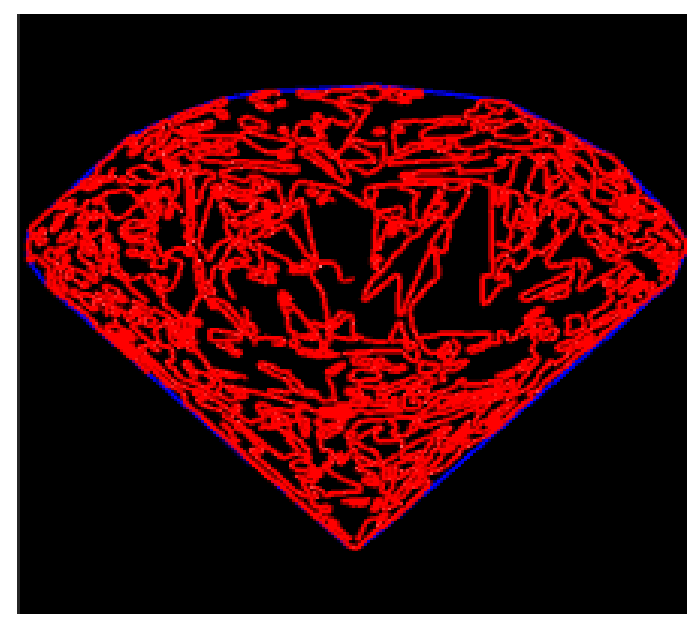

Figure 3: Shape and edge highlighted.

The edges found are higligted and the egdes are joined.

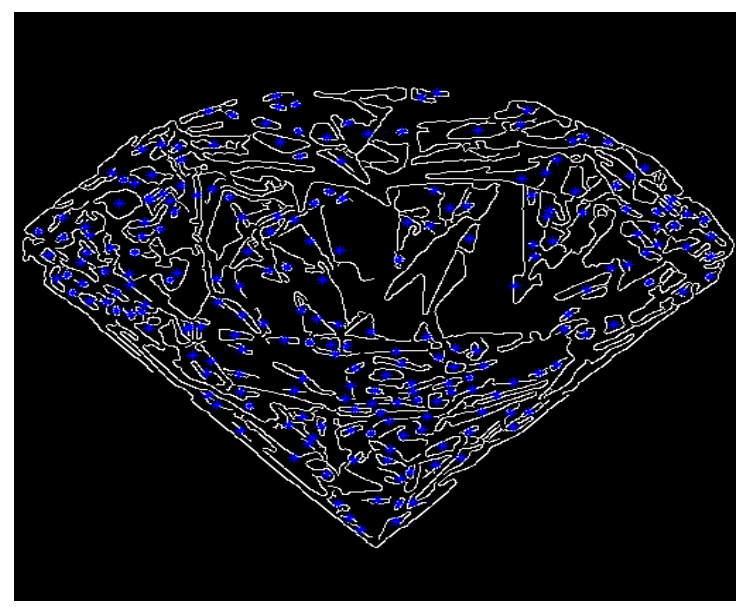

Figure 4: Points at each region.

The regions are found and maked with the blue points. The shape and the number of edges found in this type of combination is maxium.

After the edges are found the number of regions are counted and thus the number of facets are ultimately known and thus among the four C's cuts and carrot is been calculated.

After segmentation is done on the image then the features of the image is been extracted and further classification of diamond.

\section{Acknowledgement}

The authors of this paper express their gratitude to the Department of CSE, School of Engineering and 
Technology, Jain University, Bangalore for the support. The authors are also thankful to Anish Ostwal who is a diamond merchant and holds Solitaire Diamond Institute,Bangalore for giving valuable inputs for this paper.

\section{References}

1) http://www.thehindu.com/news/cities/Visakhapatn am/mobile-lab-to-check-authenticity-ofdiamonds/article7463415.ece

2) https://www.gia.edu/diamond-quality-factor

3) Jewelry Evaluation White paper. A report evaluated by enservio.

4) Prasanth.H.S, Shashidhara H.L., Balasubramanya Murthy K.N.Image Scaling Comparison Using Universal Image Quality Index. Advances in Computing, Control, \& Telecommunication Technologies, 2009. ACT '09. International Conference.

5) Olivia Mendoza, Patricia Melin, Guillermo Licea, A New Method for Edge Detection in Image Processing Using Interval Type-2 Fuzzy Logic. Granular Computing, 2007. GRC 2007. IEEE International Conference.

6) M. Mirah Kasturi , P. Sutha ,Efficient edge detection method for diagnosis of 2D and 3D lung and liver images. Inventive Systems and Control (ICISC), 2017 International Conference.

7) https://www.leaf.tv/articles/characteristics-ofdiamonds/

8) https://www.techwalla.com/articles/how-does-adiamond-tester-work

9) http://www.quicktest.co.uk/diamond-testerarticle.htm

10) http://4cs.gia.edu/en-us/diamond-clarity/

11) http://www.americangemsociety.org/page/4cs

12) https://www.lumeradiamonds.com/diamondeducation/diamond-carat-weight

13) http://www.cs.uu.nl/docs/vakken/ibv/reader/chapt er10.pdf

14) https://www.originlab.com/index.aspx?go=Produc ts/Origin/DataAnalysis/ImageProcessing

15) https://www.slideshare.net/marevishavidfather/res earch-paper-for-diamond
16) https://qz.com/854224/indias-diamond-industryis-already-weak-and-demonetization-could-pushit-into-critical-care/ article by Madhura Karnik, 7 December 2016.

17) https://www.diamondere.com/d/information/diam ond-guide 